\title{
ON THE GROUP OF REAL ANALYTIC DIFFEOMORPHISMS OF A COMPACT REAL ANALYTIC MANIFOLD \\ BY
}

\section{J. LESLIE}

\begin{abstract}
In this paper we exhibit a Lie group structure on the group of real analytic diffeomorphisms of a compact real analytic manifold. Further, we show that a variant of the Kupka-Smale theorem holds for the underlying topology of the above mentioned Lie group.
\end{abstract}

Introduction. The overall objective of this work is to prove an analytic version of the Kupka-Smale theorem: Given a smooth compact manifold, $M$, there exists a subset $R \subseteq \operatorname{Diff}^{r}(M)$ which is a countable intersection of open dense subsets of $\operatorname{Diff}^{r}(M)$ so that if $f \in R$ and $p$ is a periodic point of $f$ of order $n$ (i.e., $p \in P_{n}(f)$ ), then

(i) the eigenvalues of $T_{p} f^{n}$ lie off the unit circle, and

(ii) the stable manifold, $W^{s}(p)$, and unstable manifold, $W^{u}(p)$, through $p$ are injectively immersed; further, for $p \in P_{n}(f) W^{s}(p)$, and the unstable manifold, $W^{u}(q), q \in P_{m}(f), m$ arbitrary, intersect transversally; symbolically, $W^{s}(p) \pitchfork$ $W^{u}(q)$.

The significance of this theorem to the study of the stability problem in differential equations has been discussed by Smale [16]; this theorem has been extended to retarded functional differential equations [6].

The proofs of these theorems known to me rely heavily on transversality theory which in turn relies heavily on the implicit function theorem for Frechet differentiable functions defined in open subsets of Banach spaces. While this does not create a major theoretical difficulty in proving an analogue of the Kupka-Smale theorem for $\operatorname{Diff}^{\infty}(M)=\lim _{r} \operatorname{Diff}^{r}(M)$ because of well-known propositions concerning inverse limits, we have found that for $M$ real analytic the path for proving an analogue of the Kupka-Smale theorem is more circumlocutious. The first problem we encountered was to find an appropriate "natural" topology for $\operatorname{Diff}{ }^{\omega}(M)$; our second set of problems was the discovery of a notion of differentiability in the context of our chosen topology for which an implicit function theorem holds which would be adequate for transversality theory.

The topology that we found "natural" turned out to be equivalent to the classical $C^{\omega}$ topology studied by Van Hove [10] and J. S. E. Silva [15]; that is, an inductive limit of spaces of complex analytic functions. Colombeau [3] in an unpublished

Received by the editors January 13, 1981 and, in revised form, September 28, 1981.

1980 Mathematics Subject Classification. Primary 58F15, 58H05. 
paper has given an implicit function theorem adequate for our purposes; unfortunately, I had some difficulty with an essential point in his proof. I shall give Colombeau's theorem in a more restricted context than in his original work with a correspondingly easier proof (Theorem 3.2).

In $\$ 2$ we define Silva spaces and Silva differentiability, and give the fundamental propositions concerning Silva differentiability.

In $\S 3$ we prove the Inverse Function Theorem and the Implicit Function Theorem for Silva differentiable functions.

In $\$ 4$ we exhibit a differential structure on the group of real analytic diffeomorphisms of a compact, connected, real analytic manifold $M$. The structure in question is modelled on an inductive limit of Banach spaces. This inductive limit in general is not metrisable.

In $\S 5$ we develop the elements of transversality theory necessary for our purposes.

In $\S 6$ after having defined "generic" in this context we give some generic properties of real analytic diffeomorphisms.

\section{Silva spaces.}

Definition 2.1. Let $E_{l}, l=1,2, \ldots, n, \ldots$, be a sequence of Banach spaces and $i_{l+1}^{l}: E_{l} \rightarrow E_{l+1}$ a sequence of continuous compact injective linear operators. $E$ $=\lim _{l} E_{l}$ will be called a Silva space.

$\overrightarrow{W e}$ shall suppose for convenience that $E_{l} \subseteq E_{l+1}$ and that \|\|$_{l+1} \leqslant\|\|_{l}$. For $\varepsilon>0$ let $B_{l}^{\varepsilon}=\left\{x \in E_{l} \mid\|x\|_{l}<\varepsilon\right\}$. Let $\varepsilon_{l}>0$ be an arbitrary sequence of positive real numbers, sets of the form

$$
\bigcup_{k>0}\left(\sum_{l=1}^{k} B_{l}^{\varepsilon_{l}}\right)
$$

form a fundamental system of neighborhoods for the inductive limit topology on $E=\lim _{l} E_{l}$. Here by abuse of notation $\sum_{l=1}^{k} B_{l}^{\varepsilon_{l}} \subset \lim _{l} E_{l}$ designates the image of $\sum_{l=1}^{k} \overrightarrow{B_{l}}$ in the direct sum, $\sum_{l=1}^{\infty} E_{l}$, under the canonical map $\sum_{l=1}^{\infty} E_{l} \rightarrow \lim _{l} E_{l}$. It is immediate that

Proposition 2.1. Silva spaces are Lindelöf (i.e. every open covering contains a countable subcovering) and separable.

Proposition 2.2. A Silva space is complete.

Proof. Suppose $\mathscr{E}$ is a Cauchy filter on $E=\lim _{n} E_{n}$ which has no limit point in $E$; let $\mathcal{V}$ be the filter of neighborhoods of $0 \in E$. It follows that $\{E+V: E \in \mathcal{E}$ and $V \in \mathfrak{V}\}$ must generate a filter $\mathcal{E}^{\prime}$ which has no limit point in $E$.

For each pair of real numbers $n, k>0$ let $B_{n}^{k} \subseteq E_{n}$ be the sphere with center $0 \in E_{n}$ and radius $k$; given any $n, k>0$ there must exist $E_{n, k}^{\prime} \in \mathcal{E}^{\prime}$ so that $E_{n, k}^{\prime} \cap B_{n}^{k}=\varnothing$; for otherwise, the sets $E^{\prime} \cap B_{n}^{k}, E^{\prime} \in \mathcal{E}^{\prime}$, would form the basis of a Cauchy filter on $B_{n}^{k}$, and $B_{n}^{k}$ being relatively compact in $E$, this Cauchy filter would have a limit point $f \in E$ (see [2]); $f$ would now be a limit point of $\mathcal{E}^{\prime}$ and therefore of $\varepsilon$. 
Suppose $E_{n, k}^{\prime}=E_{n, k}+V_{n, k}, E_{n, k} \in \mathcal{E}$ and $V_{n, k} \in \mathcal{V}$. We may suppose that $V_{n, k}$ are in the form $(*)$. In particular, suppose

$$
V_{n, n}=\bigcup_{N}\left\{\sum_{m=1}^{N} B_{m}^{\varepsilon_{n, m}}\right\}
$$

where $\varepsilon_{n, m}<\varepsilon_{n-1, m} \leqslant 2^{-m}$. Set

$$
V=\bigcup_{N}\left\{\sum_{m=1}^{N} B_{m}^{\varepsilon_{m, m}}\right\} \in \mathcal{V},
$$

we have

$$
V \subset V_{n, n}+B_{n-1}^{\varepsilon_{n-1, n-1}}+\cdots+B_{1}^{\varepsilon_{1,1}} \subset V_{n, n}+B_{n}^{1}
$$

since $B_{n-1}^{\varepsilon} \subset B_{n}^{\varepsilon}$ and $\varepsilon_{n-1, n-1}+\cdots+\varepsilon_{1,1} \leqslant 1 / 2^{n-1}+\cdots+1 / 2<1$ for all $n \geqslant 2$; on the other hand, $B_{n}^{n} \cap E_{n, n}^{\prime}=\varnothing$; therefore

$$
E_{n, n} \cap\left\{B_{n}^{n}+V_{n, n}\right\}=\varnothing \text {. }
$$

We shall show that this leads to a contradiction. Note that $B_{n}^{n-1}+V \subseteq B_{n}^{n-1}+B_{n}^{1}$ $+V_{n, n} \subseteq B_{n}^{n}+V_{n, n}$.

Since $\mathcal{E}$ is a Cauchy filter there exists $E \in \mathcal{E}$ so that $f-g \in V$ for $f, g \in E$. Let $n>0$ be so that $E \cap B_{n}^{n-1} \neq \varnothing$, for $f \in E \cap B_{n}^{n-1}$, let $g \in E \cap E_{n, n}$, since $g-f \in$ $V$, we have $g \in B_{n}^{n-1}+V$ which contradicts (1) above. Q.E.D.

Given $a \in E=\lim E_{n}$, set $n(a)=\inf _{k}\left\{k: a \in E_{k}\right\}$.

Definition 2.2. Let $E=\lim _{n} E_{n}$ and $F=\lim _{n} F_{n}$ be Silva spaces and $U \subseteq E$ an open set; $f: U \rightarrow F$ is said to be Silva differentiable (resp. Silva $C^{n}$ on $U$ ) at $a \in U$ if for each $n \geqslant n(a)$ we have $m_{a}(n) \geqslant 1$ and a neighborhood $V_{n}$ of $a$ in $E_{n}$ so that $V_{n} \subseteq U$ and $\left(f \mid V_{n}\right): V_{n} \rightarrow F_{m_{a}(n)}$ is Fréchet differentiable (resp. Fréchet $C^{n}$ ) at $a$.

The elementary properties (e.g., chain rule, linearity, Taylor's expansion) follow from the analogous properties in the case of Fréchet differentiability.

In particular, we are able to show

LEMMA 2.1. If $f: U \rightarrow F$ is Silva $C^{n}$, then there exists $k$-multilinear symmetric continuous functions $D^{k} f(x): E \times \cdots \times E \rightarrow F, 1 \leqslant k \leqslant n, x \in U$, so that $D^{k} f$ : $U \times E \times \cdots \times E \rightarrow F$ are continuous and $F_{k}(v)=f(x+v)-f(x)-D f(x)(v)$ $-\cdots-\frac{1}{k !} D^{k} f(x)(v, \ldots, v), 1 \leqslant k \leqslant r$, satisfy the property that

$$
G(t, v)= \begin{cases}F(t v) / t^{k}, & t \neq 0, \\ 0, & t=0,\end{cases}
$$

is continuous at $(0, v)$.

Proof. See [4] and [13].

In [4] Colombeau has proved the following concerning Silva differentiability.

Proposition 2.3 (Colombeau). Suppose that $U, U^{\prime} \subseteq E$ are open sets in a Silva space and that $f: U \rightarrow U^{\prime}$ is a $C^{n}, n \geqslant 2$, bijection so that

(i) $f^{\prime}(x): E \rightarrow E$ is an isomorphism for $x \in U$, and 
(ii) suppose $g=f^{-1}: U^{\prime} \rightarrow U$ has the property for $y_{0} \in U^{\prime}$ and for $n \geqslant n\left(y_{0}\right)$ we have $\tau_{n}>0, K>0$, and $m(n)>0$ with $\left\|g\left(y_{0}+\sigma\right)-g\left(y_{0}\right)\right\|_{m(n)} \leqslant K\|\sigma\|_{n}$ or for $\|\sigma\|_{n}<\tau_{n}$ (uniformly Lipschitzian), and

(iii) suppose that the mapping

$$
F: x \rightarrow[D f(x)]^{-1}\{U \rightarrow L(E, E)\}
$$

has the property that for each $x_{0} \in U$ for $n \geqslant n\left(x_{0}\right)$ we have an $E_{n}$-neighborhood of $x_{0}, V_{n}\left(x_{0}\right)$, and numbers $p(l), q(l)>0$ for each $l$ so that $F\left(V_{n}\left(x_{0}\right)\right)\left(B_{l}^{1}\right) \subseteq B_{q(l)}^{p(l)}$ (locally bounded).

Then $f^{-1}$ is a $C^{n}$ function.

3. Inverse function theorem. In this section we prove an implicit theorem which is different in its hypotheses from some of the implicit function theorems inspired by Nash. References can be found in Zehnder's paper [18] for the Nash type implicit function theorem.

Definition 3.1. Let $E=\lim _{\rightarrow} E_{n}$ and $E^{\prime}=\lim _{n} E_{n}^{\prime}$ be Silva spaces and $U \subseteq E$ open. A function $f: U \rightarrow E^{\prime}$ is called admissible at $a \in U$ when there exists an auxiliary Silva space $E^{\prime \prime}=\lim _{\rightarrow} E_{n}^{\prime \prime}$, an open set $V \subseteq E^{\prime \prime}$ and a $C^{n}$ function $\tilde{f}$ : $U \times V \rightarrow E^{\prime}$ satisfying the condition that there exists a chain of bounded, closed, convex subsets

$$
B_{1} \subseteq B_{2} \subseteq \cdots \subseteq B_{n} \subseteq B_{n+1} \subseteq \cdots \subseteq E^{\prime} \times E^{\prime \prime}
$$

with $B_{i}$ a neighborhood of 0 in $E_{i}^{\prime} \times E_{i}^{\prime \prime}$, where $E^{\prime}=\lim _{1} E_{i}^{\prime}$ and $E^{\prime \prime}=\lim _{\rightarrow} E_{i}^{\prime \prime}$, so that for $\lambda>0$ there exists $\eta>0$ such that for $h \in \eta B_{i}$ we have

$$
\left(f^{\prime}(a+h)-f^{\prime}(a)\right)\left(B_{i}\right) \subseteq \lambda f^{\prime}(a)\left(B_{i}\right) \text { for all } i,
$$

and for some point $y_{0} \in V, \tilde{f}\left(x, y_{0}\right)=f(x)$ for all $x \in U$.

REMARK. The notion of admissibility arises from an effort to mimick the classical proof of the implicit function theorem (see Dieudonné, Foundations of modern analysis, Academic Press, New York, 1969, pp. 264-273) in the setting of the inductive limit. In order to keep control of the different infinite series which arise at different levels of the inductive limit a condition such as $(*)$ seems well advised. $(*)$ however is too restrictive for the applications we have in mind; we were led to the notion of admissibility by considering the relation between free and projective modules and observing that under certain conditions that the summands inherit the properties of the sum.

We now show that the class of admissible functions includes the class of $C^{2}$ functions on a Silva space with a finite dimensional range.

Proposition 3.1. Let $E=\lim _{n} E_{n}$ be a Silva space, $U \subseteq E$ open, $a \in U$, and $f$ : $U \rightarrow R$ a $C^{2}$ map so that $f^{\prime}(a) \not \overrightarrow{0}$. Then $f$ is admissible at $a \in U$.

Proof. Let $a \in U$ and $V \subseteq E$ an open neighborhood of $0 \in E$ small enough so that $a+t h \in U$ for $h \in V$ and $0 \leqslant t \leqslant 1$ and so that $D^{2} f(a+t h ; V, V) \subseteq(-b, b)$, $b \neq 0$. Suppose $V=\cup_{N}\left(\sum_{i=1}^{N} B_{i}^{\varepsilon_{i}}\right), \varepsilon_{n+1}<\varepsilon_{n}$. Let $k=\inf _{i}\left\{i: \exists \alpha \in B_{i}^{\varepsilon_{i}}\right.$ with 
$D f(a ; \alpha) \neq 0\}$, and let $\alpha_{0} \in B_{k}^{\varepsilon_{k}}$ be so that $\left|D f\left(a ; \alpha_{0}\right)\right|=r \neq 0$, we have $[-r, r] \subseteq$ $D f\left(a ; \sum_{i=1}^{N} B_{i}^{\varepsilon_{i}}\right)$ for $N \geqslant k$. Choose $\eta<\inf \{1, \lambda r / b\}$ and set $B_{l}=\cup_{N=1}^{l}\left(\sum_{i=1}^{N} B_{i}^{\varepsilon_{i}}\right)$. Q.E.D.

COROLlaRY 1. Let $E=\lim _{n} E_{n}$ be a Silva space, $U \subseteq E$ open, $a \in U$, and $f$ : $U \rightarrow R$ a $C^{2}$ map. Then $f$ is admissible.

Proof. $\tilde{f}: \quad E \times R \rightarrow R$ given by $\tilde{f}(\alpha, r)=f(\alpha)+r$ satisfies hypotheses of the proposition.

Corollary 2. Under the hypotheses of Proposition 3.1 suppose $f: U \rightarrow R^{n}$ is a $C^{2}$ map, then $f$ is admissible.

THEOREM 3.1. Let $E=\lim _{n} E_{n}$ and $F=\lim _{n} F_{n}$ be Silva spaces with $(a, b) \in E$ $\times F$ and suppose

(i) $f: E \times F \rightarrow F$ is a $C^{1}$ map so that $f(a, b)=0$ and $f_{y}(a, b) \in \operatorname{Isom}(F, F)$; where $f_{y}$ is the derivative with respect to the second variable;

(ii) $B_{n} \subseteq E_{n}$ and $C_{n} \subseteq F_{n}$ are the closed unit balls in $E_{n}$ and $F_{n}$ respectively and that there exist $\varepsilon_{0}>0, N>n(a)$ so that for each $n \geqslant N$ there exists $\tau_{n}>0$ so that

$$
f\left(a+\tau B_{n}, b\right) \subset \varepsilon_{0} \tau f_{y}(a, b) C_{n} \quad \text { if } \tau \leqslant \tau_{n} ;
$$

(iii) there exist sequences $\varepsilon_{l}>0$ and $n_{l} \leqslant M, M$ is a positive integer, so that $\Sigma_{l} \varepsilon_{l}<\infty$, and

$$
\left\{\begin{array}{l}
x \in a+\tau_{n} B_{n} \text { and } \\
y \in b+\tau\left(\varepsilon_{0} C_{n_{0}}+\varepsilon_{1} C_{n_{1}}+\cdots+\varepsilon_{l} C_{n_{l}}\right)
\end{array}\right.
$$

imply

$$
\left[f_{y}(x, y)-f_{y}(a, b)\right] C_{n_{l}} \subseteq\left(\varepsilon_{l+1} / \varepsilon_{l}\right) f_{y}^{\prime}(a, b) C_{n_{l+1}} .
$$

Set $P_{1}=\cup_{n} \tau_{n} B_{n}$.

Then there exists a function $u: a+P_{1} \rightarrow F$ so that

$$
u(a)=b, \quad f(x, u(x))=0 \quad \text { for } x \in a+P_{1},
$$

$\left(u \mid a+\tau_{n} B_{n}\right):\left(a+\tau_{n} B_{n}\right) \rightarrow F_{t(n)}$ is Fréchet differentiable for some function of $n, t(n)$, with $u^{\prime}(a)=f_{y}(a, b)^{-1} \circ f_{x}(a, b)$, here $f_{x}(a, b) \in L(E, F)$ is the derivative with respect to the first variable and lastly for $x \in a+B_{n}, y_{1}, y_{2} \in\left(b+\varepsilon_{0} C_{n_{0}}\right)$ with $f\left(x, y_{1}\right)$ $=f\left(x, y_{2}\right)=0$, we have $y_{1}=y_{2}$.

Proof. Without loss of generality we may suppose that $a=b=0$ and that $f_{y}(0,0)=I_{F}$. Set $g(x, y)=y-f(x, y)$ so that $g(x, y)=y$ if and only if $0=f(x, y)$. Now $g\left(x, y_{1}\right)-g\left(x, y_{2}\right)=y_{1}-y_{2}-f\left(x, y_{1}\right)+f\left(x, y_{2}\right) \equiv A\left(x, y_{1}, y_{2}\right)$. By a Lagrange remainder argument one has that

(I) $A\left(x, y_{1}, y_{2}\right)$ is in the convex hull of

$$
\left\{f_{y}(x, y)\left(y_{2}-y_{1}\right)-\left(y_{2}-y_{1}\right) ; y=y_{1}+t\left(y_{2}-y_{1}\right), 0 \leqslant t \leqslant 1\right\} \text {. }
$$


Given $n$ suppose $u_{0}(x)=0$ for $x \in \varepsilon_{n} B_{n}$; set $u_{1}(x)=g\left(x, u_{0}(x)\right)=-f(x, 0)$. For $0<\tau \leqslant \tau_{n}$ we have $u_{1}\left(\tau B_{n}\right) \subset \tau \varepsilon_{0} C_{n_{0}}$. Let $u_{2}(x)=g\left(x, u_{1}(x)\right)$; we have $u_{2}(x)-$ $u_{1}(x)=g\left(x, u_{1}(x)\right)-g\left(x, u_{0}(x)\right)=A\left(x, u_{1}(x), u_{0}(x)\right)$; supposing $\tau \leqslant \tau_{n}$ and $x \in$ $\tau B_{n}$ we have $u_{1}(x)-u_{0}(x) \in \tau \varepsilon_{0} C_{n_{0}}$; from (I) and (*) we have $u_{2}(x)-u_{1}(x) \in$ $\tau \varepsilon_{1} C_{n_{1}}$. By iteration setting $u_{l+1}(x)=g\left(x, u_{l}(x)\right)$, we have $u_{l+1}(x)-u_{l}(x) \in \tau \varepsilon_{l} C_{n_{l}}$; since $\sum \varepsilon_{n}<\infty, n_{l} \leqslant M$ for all $l$, and $F$ is complete, setting $P_{1}=\cup_{n} \tau_{0} B_{n}$ we may define $u(x) \equiv \lim _{n} u_{n}(x)$. As $g$ is continuous we have $u(x)=g(x, u(x))$ so that $f(x, u(x))=0$. Since $u(x) \in \tau\left(\left(\sum \varepsilon_{l}\right) C_{M}\right)$, it follows that $u(x)$ is uniformly Lipschitzian (see Proposition 2.3(ii)) and therefore differentiable at 0 with $u^{\prime}(0)=$ $-f_{y}(0,0)^{-1} \circ f_{x}(0,0)$ since

$$
\begin{aligned}
f_{x}(0,0) h+f_{y}(0,0) u(h)= & \underbrace{-\left[f(h, u(h))-f(h, 0)-f_{y}(0,0) u(h)\right]}_{S_{1}} \\
& \underbrace{-\left[f(h, 0)-f(0,0)-f_{x}(0,0) h\right]}_{S_{2}}
\end{aligned}
$$

and there exists $N$ so that $\lim _{|h|_{n(h)} \rightarrow 0}\left|S_{i}\right|_{N} /|h|_{n(h)}=0$.

(II) Given $x \in B_{n} ; y_{1}, y_{2} \in C_{n_{0}}$ so that $f\left(x, y_{1}\right)=f\left(x, y_{2}\right)=0 ; y_{1}-y_{2}=g\left(x, y_{1}\right)$ $-g\left(x, y_{2}\right)=A\left(x, y_{1}, y_{2}\right) \in 2 \varepsilon_{1} C_{n_{1}}$ by iteration for each $l, y_{1}-y_{2} \in 2 \varepsilon_{l} C_{n_{1}}$, therefore $y_{1}=y_{2}$.

Let us recall that in a topological vector space $E$ a subset $D$ centered at $a \in Z$ is called bornivorous when $D-a$ absorbs every bounded set $B \subseteq E$; that is, there exists $\lambda>0$ with $\lambda B \subseteq D-a$. In [9, Chapter 7, Proposition 2, p. 61] Hogbe-Nlend proves

Proposition 3.2. If a subset $U$ of a Silva space $E$ has the property that every $x \in U$ is the center of a bornivorous subset $D_{x} \subseteq U$, then it is an open set.

Theorem 3.2. Suppose $E=\lim _{n} E_{n}$ is a Silva space, $U \subseteq E$ open, and suppose $f$ : $U \rightarrow E$ an admissible $C^{1}$ map with $f^{\prime}(a): E \rightarrow E$ an automorphism. Then there exist open neighborhoods $U_{1} \subseteq U$ of $a$ and $V_{1}$ of $f(a)$ so that $f \mid U_{1}$ is an isomorphism from $U_{1}$ onto $V_{1}$; further, $f^{-1}: V_{1} \rightarrow U_{1}$ is admissible.

Proof. Without loss of generality we may supppose $f$ a $C^{1}$ map satisfying (*) of Definition 3.1, since $g: E \times E^{\prime \prime} \rightarrow E \times E^{\prime \prime}$ given by $g(x, y)=(\tilde{f}(x, y), y)$ would satisfy $(*)$ and the hypothesis of the theorem at $\left(x_{0}, y_{0}\right) . g$ being a local isomorphism at $\left(x_{0}, y_{0}\right)$ would imply that $f$ is a local isomorphism at $x_{0}$. Suppose $a=f(a)=0$ and suppose $f^{\prime}(a)=\mathrm{id}_{E}$ which we may do without a loss of generality. Now for $0<\lambda<1$ let $\eta>0$ be so that for $h \in \eta B_{i}$ we have $\left(f^{\prime}(a+h)-\mathrm{id}_{B_{i}}\right)\left(B_{i}\right) \subseteq \lambda B_{i}$ for all $i \geqslant \sup \left\{\inf _{k}\left(k: a \in E_{k}\right), \inf _{l}\left(l: h \in E_{l}\right)\right\}$. It follows that $f^{\prime}(a+h)$ is an automorphism for $h \in Q=\cup_{i} \eta B_{i}$. Note that $Q$ is a bornivorous subset centered at 0 . By Proposition 3.2 $U=\left\{x \in E: f^{\prime}(x) \in \operatorname{Aut}(E)\right\}$ is an open neighborhood of 0 . Note also that the hypotheses on $f$ imply that $f^{\prime}: U \times E \rightarrow E$ is continuous. Define $F$ : $E \times U \rightarrow E$ by $F(y, x)=f(x)-y$. As $F_{x}(0,0)=\mathrm{id}_{E}$ the hypotheses of Theorem 3.1 are easily seen to be verified; therefore, we have that there exists a bornivorous subset centered at $0=f(0), D$, and a $C^{1}$ mapping $u: D \rightarrow U$ so that $f(u(x))=x$; 
further $u^{\prime}(0)=f^{\prime}(0)^{-1} \equiv$ identity on $E$. Mutatis mutandis the same argument holds for all $a \in U$; therefore, by Proposition 3.2, the image of $f \mid U$ contains a neighborhood of $b, V$, and thus $f: U \rightarrow E$ is an open mapping. Now we shall verify that there exists a neighborhood of 0 on which $f$ is injective. This is proved by induction. By classical arguments concerning the Fréchet derivative there exists $\varepsilon_{n(a)}>0$ so that $f \mid B_{n(a)}^{\varepsilon_{n(a)}}$ is a diffeomorphism onto an open set in $E_{n(a)}$. Suppose we have $B=B_{n(a)}^{\varepsilon_{n(a)}}$ $+B_{n(a)+1}^{\varepsilon_{n(a)+1}}+\cdots+B_{n(a)+k}^{\varepsilon_{n(a)+k}}$ so that $f \mid B$ is injective. It suffices to show that there exists $\varepsilon_{n(a)+k+1}>0$ so that $f \mid B+B_{n(a)+k+1}^{\varepsilon_{n(a)+1}}$ is injective. Let $B_{\varepsilon}=\{x \in$ $\left.E_{n(a)+k+1}: d(B, x)<\varepsilon\right\}$, where $d($,$) is the canonical metric in E_{n(a)+k+1}$. Suppose now for each $n>0$ that there exists $x_{n}, y_{n} \in B_{1 / n}$ with $x_{n} \neq y_{n}$ and $f\left(x_{n}\right)=f\left(y_{n}\right)$. Let $x_{n}$ be a subsequence of $x_{n}$ converging for the $E_{n(a)+k+2}$ topology to a point in $B$ (let us recall $B$ is compact for the induced $E_{n(a)+k+2}$ topology) and choose a subsequence of $y_{n_{l}}$ converging to a point $y \in B$. After reindexing we have converging sequences $\left\{x_{n}\right\}$ and $\left\{y_{n}\right\}$ so that $x_{n} \neq y_{n}, x_{n}, y_{n} \in E_{n(a)+k+1}, f\left(x_{n}\right)=f\left(y_{n}\right), \lim _{n} x_{n}$ $=x, \lim _{n} y_{n}=y \in B$. Therefore $f(x)=\lim _{n} f\left(x_{n}\right)=\lim _{n} f\left(y_{n}\right)=f(y)$. Since $f$ is injective on $B$ we have $x=y$; however, in every $E_{n(a)+k+2}$ neighborhood of $x=y$ we have both $x_{n}$ and $y_{n}$ for $n$ large enough so that $f\left(x_{n}\right)=f\left(y_{n}\right)$, which contradicts that $f$ is a local $E_{n+k+2}$ diffeomorphism at $x . f^{-1}$ is admissible follows from the observation that given an isomorphism $u_{0}: E_{1} \rightarrow E_{2}$ between Silva spaces satisfying (*) of Definition 3.1 and $0<k<1$ that $\left(u-u_{0}\right)\left(B_{i}^{1}\right) \subseteq k u_{0}\left(B_{i}^{1}\right)$ would imply $\left(u^{-1}-u_{0}^{-1}\right) u_{0}\left(B_{i}\right) \subseteq k B_{i} /(k-1)$ and the observation that $D f^{-1}(f(x))=D f(x)^{-1}$. Q.E.D.

4. Lie group structure for the group of analytic diffeomorphisms of a compact analytic manifold. In this section we shall exhibit a differential structure on the group of analytic diffeomorphisms (see Theorem 4.1). The methods we use are essentially the same as the ones we used in [12].

Let $M^{l}$ and $N^{d}$ be compact connected real analytic manifolds. For $f \in C^{\omega}(M, N)$ $=$ the set of all real analytic functions from $M$ to $N$. Let $C_{f}^{\omega}(M, T(N))$ be the vector space of all real analytic liftings of $f$; that is, $g \in C_{f}^{\omega}(M, T N)$ is a real analytic function $g: M \rightarrow T N$ such that $\pi \circ g=f$ where $\pi: T N \rightarrow N$ is the canonical projection. Cover $N$ by a finite collection of trivializing normal relatively compact open charts $V_{i}, i=1, \ldots, n$, and $M$ by a finite collection of trivializing relatively compact open charts $U_{i}, i=1, \ldots, m$, such that $\operatorname{diam}\left(f\left(U_{i}\right)\right)<\lambda / 3$, where $\lambda$ is the Lebesgue number of $\left\{V_{j}\right\}$. Define a topological structure on $C_{f}^{\omega}(M, T N)$ as follows: let $k_{i}$ : $U_{i} \rightarrow U_{i}^{\prime} \subset R^{l}$ and $l_{j}: V_{j} \rightarrow V_{j}^{\prime} \subseteq R^{d}$ be homeomorphisms determining the local real analytic structure of $M$ and $N$ respectively. Suppose $f\left(U_{i}\right) \subset V_{j(i)}$. Let $\phi_{j(i)}: \pi^{-1}\left(V_{j(i)}\right)$ $\rightarrow V_{j(i)}^{\prime} \times R^{d}$ be a real analytic diffeomorphism.

By a uniformly bounded analytic function we intend an analytic function, $f(x)$, so that there exists $l$ and $M$ with

$$
\left\|f^{(k)}(x)\right\| \leqslant k ! M l^{k} \quad \text { for all } x .
$$

Suppose $C_{b}^{\omega}\left(U_{i}^{\prime}, R^{d}\right)$ is the vector space of uniformly bounded real analytic maps $f: U_{i}^{\prime} \rightarrow R^{d}$; as a vector space $C_{b}^{\omega}\left(U_{i}^{\prime}, R^{d}\right)=\lim E_{l}$, where $E_{l}\left(U_{i}^{\prime}, R^{d}\right)$ is the Banach 
space of uniformly bounded real analytic functions $f: U_{i}^{\prime} \rightarrow R^{d}$ so that $\|f(x)\|_{l} \leqslant$ $k ! p l^{k}$ for some $p$, we set $\|f(x)\|_{l}=\inf _{p}\left\{p:\|f(x)\|_{l} \leqslant k ! p l^{k}\right.$ for all $\left.x \in U_{i}^{\prime}\right\}$. The maps $E_{l} \rightarrow E_{l+1}$ are the canonical injections. We shall consider $C_{b}^{\omega}\left(U, R^{d}\right)$ for the rest of our paper with the above inductive limit topology. We call this topology on $C_{b}^{\omega}\left(U_{i}^{\prime}, R^{d}\right)$ the Van Hove topology.

Designate by $B_{k}^{p}\left(U, R^{m}\right) \subseteq E_{k}\left(U, R^{m}\right)$ the ball of radius $p$ centered at the origin in $E_{k}$.

LEMma. $C_{b}^{\omega}\left(U, R^{m}\right)$, where $U \subseteq R^{n}$ is open, is a Silva space, if $U$ is of finite diameter.

Proof. Let $a_{1}, \ldots, a_{n}, \ldots \in B_{k}^{p}\left(U, R^{m}\right)$ and let $a_{1}^{(s)}, \ldots, a_{n}^{(s)}, \ldots$ be a subsequence so that $\left\|D^{i} a_{l / y}^{(s)}-D^{i} a_{l / z}^{(s)}\right\|_{k^{\prime}} \leqslant \frac{1}{s}$ for $i \leqslant \log (2 s p) /\left(\log \left(k^{\prime}\right)-\log (k)\right), s$ a positive integer. This is possible by the Weierstrass argument since $B_{k}^{p}\left(U, R^{m}\right)$ is uniformly equicontinuous on $U$. The above implies that $a_{1}^{(s)}, \ldots, a_{n}^{(s)}, \ldots$ is a $\frac{1}{s}$-net in $B_{k^{\prime}}^{p}$. Now, by Cantor diagonalization we obtain a Cauchy subsequence in $E^{k^{\prime}}\left(U, R^{m}\right)$. Q.E.D.

Set $\mathcal{Q}_{0}=\sum_{i=1}^{n} C_{b}^{\omega}\left(U_{i}^{\prime}, R^{d}\right)$, with the canonical direct sum topology $\mathcal{Q}_{0}$ is a Silva space. Define $\gamma: C_{f}^{\omega}(M, T N) \rightarrow \mathbb{Q}_{0}$ by $\gamma(g)=g_{0}+\cdots+g_{m}$ where $g_{i} \in C^{\omega}\left(U_{i}^{\prime}, R^{d}\right)$ is the composite

$$
U_{i}^{\prime} \stackrel{k_{i}^{-1}}{\rightarrow} U_{i} \stackrel{g}{\rightarrow} \pi^{-1}\left(V_{j(i)}\right) \rightarrow V_{j(i)}^{\prime} \times R^{d^{\text {proj }}} \rightarrow R^{d}
$$

Let $\mathbb{Q}=\gamma\left(C_{f}^{\omega}(M, T N)\right) \subseteq \mathbb{Q}_{0}$; $\mathbb{Q}$ is a closed subspace $\mathbb{Q}_{0}$ and therefore the induced topology on $\mathbb{Q}$ is a Silva topology. By means of $\gamma$ we transport the induced Silva structure to $C_{f}^{\omega}(M, T N)$.

When $M=N$ and $f=\mathrm{id}_{M}, \mathbb{Q}=\Gamma^{\omega}(M)$ is the vector space of real analytic vector fields on $M$.

From the Cauchy inequalities and classical theorems on inductive limits we obtain

LEMMA 4.1. Suppose $M$ analytically embedded in $R^{N}$ (Jacobowitz [11]), $R^{N}$ canonically embedded in $C^{N}, \quad U_{i}=\left\{x \in M^{C}: d(x, M)<\frac{1}{i}\right.$, where $d\left(\left(x_{1}, \ldots, x_{n}\right)\right.$, $\left.\left(z_{1}, \ldots, z_{n}\right)\right)=\max _{i}\left(\left|x_{i}-z_{i}\right|\right)$ and where $M^{C}$ is the Whitney-Bruhat complexification of $M$ \}, and suppose $F_{i}$ is the vector space of all bounded complex analytic vector fields on $U_{i}$ which extend real analytic vector fields on $M$. Then $\lim F_{i} \approx$ the $C^{\omega}$ topology on the vector space of real analytic vector fields on $M, \Gamma^{\omega}(M)$, where the norm on $F_{i}$ is the sup norm.

REMARK 4.1. The topology on $\Gamma^{\omega}(M)$ is independent of the charts $\left(U_{i}, k_{i}\right)$ and $\left(V_{j}, l_{i}\right)$.

When we consider a Riemannian manifold $N$ as a metric space $(N, p)$ it will always be with respect to the metric $p$ determined by the Riemannian structure.

Description of a "canonical" chart at $f \in C^{\omega}(M, N)$. Let $\lambda$ be the Lebesgue number of the covering $V_{j}$. For $y \in C^{\omega}(M, N)$ such that $p(f(s), y(s))<\lambda / 3$ for all $s \in M$ set $\alpha(s)=\exp _{f(s)}^{-1}(y(s)) ; \alpha \in C_{f}^{\omega}(M, T N)$; let $U_{f}=\left\{y \in C^{\omega}(M, T N) \mid\right.$ $p(y(s), f(s))<\lambda / 3$ for all $s \in M\}, \psi_{f}: U_{f} \rightarrow C_{f}^{\omega}(M, T N)$ given by $\psi_{f}(y)=\alpha$ is taken as a chart of $C^{\omega}(M, N)$ at $f$. 
Proposition 4.1. If $U_{x}$ and $U_{x^{\prime}}$ are overlapping coordinate charts of $x, x^{\prime} \in$ $C^{\omega}(M, N)$, then the coordinate transformation

$$
\psi=\psi_{x^{\prime}} \circ \psi_{x}^{-1}: \psi_{x}\left(U_{x} \cap U_{x^{\prime}}\right) \rightarrow \psi_{x^{\prime}}\left(U \cap U^{\prime}\right)
$$

is a Silva $C^{\infty}$ transformation.

In order to prove the above proposition we shall need the following two lemmas.

LEMMA 4.2. Let $U, U^{\prime} \subseteq R^{n}$ be open subsets, and $B_{l}^{k}\left(U, U^{\prime}\right)=\left\{h: U \rightarrow U^{\prime}, h\right.$ analytic with $\left.\left\|D^{n} h(x)\right\| \leqslant n ! k l^{n}, x \in U\right\}$, and $B_{l^{\prime}}^{k^{\prime}}\left(U^{\prime}, R^{n}\right)=\left\{h: U^{\prime} \rightarrow R^{n}, h\right.$ analytic with $\left.\left\|h^{(n)}(x)\right\| \leqslant n ! k^{\prime}\left(l^{\prime}\right)^{n}, x \in U^{\prime}\right\}$. If $g \in B_{l^{\prime}}^{k^{\prime}}\left(U^{\prime}, R^{n}\right)$ and $f \in B_{l}^{k}\left(U, U^{\prime}\right)$, then $(g \circ f) \in B_{2 l\left(1+k l^{\prime}\right)}^{k^{\prime}}\left(U, R^{n}\right)$.

ProOF.

$$
D^{t}(g \circ f)=\sum_{1 \leqslant q \leqslant t} \sum_{i_{1}+\cdots+i_{q}=t} \sigma_{t}\left(i_{1}, \ldots, i_{q}\right) D^{q} g\left(f(x) ; D^{i_{1}} f(x), \ldots, D^{i_{q}} f(x)\right)
$$

where the $\sigma_{t}$ are defined iteratively by $\sigma_{k}(k)=1, k \geqslant 1$, and with $\sum_{j=1}^{q-1} i_{j}=k, q>1$, $i_{q}=t-k, t>k$, we set

$$
\sigma_{t}\left(i_{1}, \ldots, i_{q}\right)=\left(\begin{array}{c}
t-1 \\
k
\end{array}\right) \sigma_{k}\left(i_{1}, \ldots, i_{q-1}\right)
$$

with $i_{1}, \ldots, i_{q}>0$, so that

$$
\begin{aligned}
\left\|D^{t}(g \circ f)\right\| & \leqslant \sum_{1 \leqslant q \leqslant t} \sum_{i_{1}+\cdots+i_{q}=t} q ! i_{1} ! \cdots i_{q} ! k^{\prime}\left(l^{\prime}\right)^{q} k^{q} l^{t} \sigma_{t} \\
& =(l)^{t} k^{\prime} \sum_{1 \leqslant q \leqslant t i_{1}+\cdots+i_{q}=t} \sum_{l} q ! i_{1} ! \cdots i_{q} !\left(l^{\prime} k\right)^{q} \sigma_{t}\left(i_{1}, \ldots, i_{q}\right) .
\end{aligned}
$$

Consider

$$
\begin{aligned}
& q ! i_{1} ! \cdots i_{q} ! \sigma_{t}\left(i_{1}, \ldots, i_{q}\right)=q ! i_{1} ! \cdots i_{q} ! \prod_{s=1}^{q}\left\{\frac{\left[\left(\sum_{j=1}^{s} i_{j}\right)-1\right] !}{\left(i_{s}-1\right) !\left(\sum_{j=1}^{s-1} i_{j}\right) !}\right\} \\
& =q ! i_{1} \cdots i_{q} \prod_{s=1}^{q}\left\{\frac{\left[\left(\sum_{j=1}^{s} i_{j}\right)-1\right] !}{\left(\sum_{j=1}^{s-1} i_{j}\right) !}\right\}=q ! i_{1} \cdots i_{q} \frac{\left[\left(\sum_{j=1}^{q} i_{j}\right)-1\right] !}{\prod_{s=1}^{q}\left(\sum_{j=1}^{s-1} i_{j}\right)} \\
& =q ! i_{1} \cdots i_{q} \frac{(t-1) !}{\prod_{s=1}^{q}\left(\sum_{j=1}^{s-1} i_{j}\right)}=t ! \frac{q ! i_{1}, \ldots, i_{q}}{\prod_{s=1}^{q}\left(\sum_{j=1}^{s} i_{j}\right)} .
\end{aligned}
$$

It therefore suffices to note that

$$
\sum_{i_{1}+\cdots+i_{q}=t} \frac{q ! i_{1} \cdots i_{q}}{\prod_{s=1}^{q}\left(\sum_{j=1}^{s} i_{j}\right)} \leqslant \frac{2^{t} t !}{q !(t-q) !},
$$

since each summand is $\leqslant 2^{t}$. 
Lemma 4.3. Let $C_{b}^{\omega}\left(U^{\prime}, E_{3}\right) \subseteq C^{\omega}\left(U^{\prime}, E_{3}\right)$ (resp. $\left.C_{b}^{\omega}\left(U, U^{\prime}\right) \subseteq C^{\omega}\left(U, U^{\prime}\right)\right)$ be the subspace of uniformly bounded analytic functions defined on $U$. Now suppose $\alpha \in$ $C_{b}^{\omega}\left(U^{\prime}, E_{3}\right)$ and define $F_{\alpha}: C_{b}^{\omega}\left(U, U^{\prime}\right) \rightarrow C_{b}^{\omega}\left(U, E_{3}\right)$ by $F_{\alpha}(\phi)=\alpha \circ \phi$. Then $F_{\alpha}$ is in $C^{\infty}\left(C_{b}^{\omega}\left(U, U^{\prime}\right), C_{b}^{\omega}\left(U, E_{3}\right)\right)$ and $\alpha \times \phi \rightarrow \alpha \circ \phi$ induces a $C^{\infty}$ map from $C_{b}^{\omega}\left(U^{\prime}, E_{3}\right) \times$ $C_{b}^{\omega}\left(U, U^{\prime}\right) \rightarrow C_{b}^{\omega}\left(U, E_{3}\right)$. (Note that $C_{b}^{\omega}\left(U, U^{\prime}\right)$ is open in $C_{b}^{\omega}\left(U, E_{2}\right)$ when $U^{\prime}$ is open in $E_{2}$ where $E_{2}$ and $E_{3}$ are arbitrary Euclidean spaces.)

Proof. Suppose $\alpha \in E_{l}\left(C_{b}^{\omega}\left(U^{\prime}, E_{3}\right)\right)$, then $F_{\alpha}\left(B_{l^{\prime}}^{k^{\prime}}\left(U, U^{\prime}\right)\right) \subseteq B_{2 l^{\prime}\left(1+k^{\prime} l\right)}\left(U, E_{3}\right)$; setting $\lambda=l^{l^{n}}$ we have

$$
D^{(n)} \alpha \in B_{l^{\prime \prime}}^{\|\alpha\|_{\lambda}}\left(U^{\prime}, L_{s}^{n}\left(E_{2} ; E_{3}\right)\right),
$$

for $l^{\prime \prime}>l$ sufficiently large, where $L_{s}^{n}\left(E_{2} ; E_{3}\right)$ is the Banach space of $n$ symmetric multilinear maps $E_{2} \times \cdots \times E_{2} \rightarrow E_{3}$ with the operator norm. This last remark can easily be seen by taking $l^{\prime \prime}$ large enough so that $((n+m) ! / m !)\left(l / l^{\prime \prime}\right)^{m} \leqslant 1$ for all $m$. Now suppose $f(x) \in B_{l^{\prime}}^{k^{\prime}}\left(U, U^{\prime}\right)$ and $h(x) \in E_{l^{\prime}}\left(U, E_{2}\right)$, consider $H(x)=$ $D^{(n)} \alpha(f(x) ; h(x), \ldots, h(x))$. Note that $K=D^{(n)}(\alpha) \circ f: U \rightarrow L_{s}^{n}\left(E_{2}, E_{3}\right)$ is in

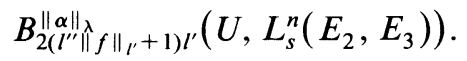

As $L_{s}^{n}\left(E_{2}, E_{3}\right) \times E_{2} \times \cdots \times E_{2} \rightarrow E_{3}$ is a continuous $n+1$ multilinear map, we obtain by a generalized Leibnitz rule that $H(x)$ is in $B_{2\left(l^{\prime \prime}\left\|_{f}\right\| \|_{l^{\prime}}+(n+1)\right) l^{\prime}}^{n}$. Now by an easy Taylor series argument we have that $F_{\alpha}$ is a $C^{n-1}$ mapping from $B_{l^{\prime}}^{k^{\prime}}\left(U, U^{\prime}\right)$ into $E_{2\left(l^{\prime \prime} k^{\prime}+(n+1)\right) l^{\prime}}\left(U, E_{3}\right)$. Thus $F_{\alpha}$ is $C^{\infty}$.

Proof of Proposition 4.1. $\psi(v)(s)=\exp _{x^{\prime}(s)}^{-1}(\exp (v(s)))$. It is classical that exp: $T(N) \rightarrow N$ is a real analytic map. Let $g$ be the Riemannian metric on $N$, set $S=\{\alpha \mid g(\alpha, \alpha)<\lambda / 3\},(\pi \times \exp ): S \rightarrow N \times N$ is an analytic diffeomorphism onto the neighborhood $U$ of the diagonal $\Delta \subset N \times N$ made up of the pairs $x, y$ with $p(x, y)<\lambda / 3$, its inverse $\phi: U \rightarrow S$ is given by $\phi(v, m)=\exp _{v}^{-1}(m)$. Set $X(s)=$ $\left(\pi^{-1}(x / s)\right) \cap S . \phi\left(x^{\prime}(s), \exp (v)\right), v \in X(s)$, defines an analytic function from $X(s)$ to $\pi^{-1}\left(X^{\prime}(s)\right)$; by Lemma 2 we have that $\psi$ is $C^{\infty}$. Q.E.D.

REMARK 4.2. The compactness of $M$ is essential to the above proof in order for the maps in question to be uniformly bounded as analytic maps, $N$ being compact is not essential.

Remark 4.3. The differential structure on $C^{\omega}(M, N)$ does not depend on the choice of Riemannian structures on $M$ or $N$, see Remark 4.1.

Lemma 4.2 also implies

Proposition 4.2. Let $M, N$, and $P$ be compact, connected, real analytic manifolds. Then composition $C^{\omega}(M, N) \times C^{\omega}(N, P) \rightarrow C^{\omega}(M, P)$ defines a $C^{\infty}$ function.

In [12] we defined a differential structure for the set of $C^{\infty}$ mappings of a compact manifold $M$ into a compact manifold $N$ that we shall designate by $C^{\infty}(M, N)$. That manifold was defined analogously to the one we are discussing here, but was modelled on the $C^{\infty}$ vector fields with the $C^{\infty}$ topology. As the $C^{\infty}$ topology induces a topology on $C_{f}^{\omega}(M, T N)$ so that open sets are bornivorous when centered at each of their points, it is a coarser topology than the Van Hove topology on $\left(C_{f}^{\omega}(M, T N)\right)$ (see Proposition 3.2); thus 
Proposition 4.3. The canonical injection $C^{\omega}(M, T N) \rightarrow C^{\infty}(M, T N)$ is a $C^{\infty}$ injection of the first manifold into the second and induces the canonical injection of the tangent bundle of the first into that of the second.

COROLlARY. Let $M$ be a compact connected analytic manifold. Then there exists an open neighborhood of the identity in $C^{\omega}(M, M)$ consisting entirely of diffeomorphisms.

Proposition 4.4. $x \rightarrow x^{-1}$ is $C^{\infty}$.

Proof. It suffices to prove that the inverse operation is smooth in some small symmetric open neighborhood of the identity. Let $U$ be an open symmetric chart at the identity and $V$ a neighborhood of the identity so that $V^{2} \subseteq U$.

Set $C_{\mathrm{id}}^{\omega}(M, T M)=T$ (id). Suppose $\tilde{V}=\psi_{e}(V)$ where $\left(V, \psi_{e}\right)$ is the canonical chart described above and suppose $\rho: \tilde{V} \rightarrow \tilde{V}$ is determined by $x \rightarrow x^{-1}$. $\rho$ is uniformly Lipschitzian at 0 ; that is, given any bounded set $B_{1} \subseteq T$ (id), there exist $\tau_{0}>0$ and a bounded set $B_{2} \subseteq T$ (id) so that $\rho\left(\tau B_{1}\right) \subseteq \tau B_{2}$ for $\tau \leqslant \tau_{0}$. To see this; suppose $f \in V$ is in $B_{l}^{k}\left(U, U^{\prime}\right)$. To simplify we suppose $0 \in U$ and $f(0)=0$ and suppose $f(\alpha)=$ $\sum \frac{1}{n !} D^{n} f(0 ; \alpha, \ldots, \alpha), \alpha \in U \cap S_{1 / l}(0)$. Then $f^{-1}(\beta)=\Sigma_{m \geqslant 1} B_{m}(\beta, \ldots, \beta)$, where $B_{m}$ is an $m$-multilinear symmetric continuous form $R^{m} \times \cdots \times R^{m} \rightarrow R^{m}$. Set $A_{n}$ $=\frac{1}{n !} D^{n} f$. As

$$
f f^{-1}(x)=x
$$

we obtain the iterative system of operator equations for $B_{i}$.

$$
A_{1} B_{n}=P_{n}\left(A_{2}, \ldots, A_{n}, B_{1}, \ldots, B_{n-1}\right),
$$

which can be calculated by means of $(*)$.

Note that $P_{n}$ has nonnegative integral coefficients, is linear in the $A_{j}$ 's and $B_{1}=A_{1}^{-1}$. Now setting $a_{i}=\left\|A_{i}\right\|$, the corresponding polynomial

$$
P_{n}\left(-a_{2}, \ldots,-a_{n}, b_{1}, \ldots, b_{n-1}\right)=b_{n} \geqslant\left\|B_{n}\right\| \sum_{i=1}^{\infty} b_{i} z^{i}=h(z)
$$

determines the inverse function to $a_{1}(z)-\sum_{i=2}^{\infty} a_{i} z^{i}$ and has a positive radius of convergence, say $\rho$. Suppose $0<\rho^{\prime}<\rho$ and $M=h\left(\rho^{\prime}\right)$, so that

$$
f^{-1} \in B_{\left(\rho^{\prime}\right)}^{M}-1 \text {. }
$$

As $t \rightarrow(1+t)^{-1}$ is Lipschitzian for $t$ small and $P_{n}\left(\lambda A_{2}, \ldots, \lambda A_{n}, B_{1}, \ldots, B_{n}\right)=$ $\lambda P_{n}\left(A_{2}, \ldots, A_{n}, B_{1}, \ldots, B_{n}\right)$ we have that $f \rightarrow f^{-1}$ is uniformly Lipschitzian at identity in Diff ${ }^{\omega}(M)$. This implies by Proposition 2.3 that $x \rightarrow x^{-1}$ is differentiable at the identity.

To prove that $x \rightarrow x^{-1}$ is $C^{\infty}$ at the identity, consider $f: V \times V \rightarrow U \times U$ given by $f(x, y)=(x, x y)$; it suffices by Proposition 2.3 to show that $f^{\prime}(x, y)^{-1}$ is a locally bounded map from $V \times V$ into $L\left(T\right.$ (id) $\times T$ (id), $T$ (id) $\times T($ id $)$ ). Now $f^{\prime}(x, y)(\alpha, \beta)$ can be represented locally as $(\alpha, D x(\beta)+\alpha \circ y)$ so that $f^{\prime}(x, y)^{-1}(\alpha, \beta)=$ $\left(\alpha, D x^{-1}(\beta)-D x^{-1}(\alpha \circ y)\right)$. As $(a, b) \rightarrow D x(a, b)$ is locally bounded and $D x \rightarrow$ $D x^{-1}$ is an analytic map from an open subset of $L\left(R^{n}, R^{n}\right)$ to $L\left(R^{n}, R^{n}\right)$ we may conclude that $\left(f^{\prime}(x, y)\right)^{-1}$ is locally bounded (see Proposition 2.3). Q.E.D. 
REMARK 4.4. In this proof one uses the manifold structure several times in order to stay in $T(\mathrm{id})$ (see [12, Proposition 7, p. 269]).

In summary, in this section we have shown

THEOREM 4.1. Given a compact, connected, real analytic manifold $M$, Diff ${ }^{\omega}(M) \equiv$ the group of real analytic diffeomorphisms with the Van Hove-Silva topology is a $C^{\infty}$ group in the sense of Silva differentiation; that is, it is a $C^{\infty}$ manifold modelled on its Silva space of real analytic vector fields, and multiplication and inversion define Silva- $C^{\infty}$ maps

$$
\operatorname{Diff}^{\omega}(M) \times \operatorname{Diff}^{\omega}(M) \rightarrow \operatorname{Diff}^{\omega}(M) \text { and } \operatorname{Diff}^{\omega}(M) \rightarrow \operatorname{Diff}^{\omega}(M) .
$$

5. A transversality theorem. In this section we prove a transversality theorem à la Abraham [1] in the context of Silva manifolds of analytic maps.

Theorem 5.1. Let $E$ and $F$ be Silva spaces, $U \subset E$ open, and suppose $f: U \rightarrow F$ is a $C^{r}(r \geqslant 2)$ admissible Fredholm map (see Definition 3.1); that is the $\operatorname{ker}(D f(x))$ and $\operatorname{coker}(D f(x))$ are finite dimensional for $x \in U$. Then for $a \in U$ there exist subspaces $E_{1}, E_{2}$, and $F_{1}$, where $E_{2}$ and $F_{1}$ are finite dimensional, open neighborhoods $U$ of $a$ in $E$ and $V$ of $f(a)$ in $F$, and $C^{r}$ maps $\alpha: U \rightarrow E_{1} \times E_{2}$ and $\beta: V \rightarrow F_{1} \times E_{1}$ (where $E_{1} \oplus E_{2}=E$ and $F_{1} \oplus E_{1}=F$ ) such that

(1) $\alpha(a)=(0,0) \in E_{1} \times E_{2} ; f(U) \subseteq V ; \alpha$ maps $U C^{r}$ diffeomorphically into $B_{1} \times$ $B_{2}$ where $B_{1}$ and $B_{2}$ are open neighborhoods of 0 in $E_{1}$ and $E_{2}$ respectively;

(2) $\beta(f(a))=(0,0) \in F_{1} \times E_{1} ; \beta$ maps $V C^{r}$ diffeomorphically onto an open subset of $F_{1} \times E_{1}$.

Finally, the local representative $\beta \circ f \circ \alpha^{-1} ; \alpha(U) \rightarrow \beta(V)$ of $f$ has the form

$$
\beta \circ f \circ \alpha^{-1}(u, v)=(\eta(u, v), u)
$$

for $u \in B_{1}, v \in B_{2}$ where $D \eta(0,0)=0$.

Proof. By the Hahn-Banach theorem we may assume $E=E_{1} \times E_{2}$ and $F=F_{1}$ $\times E_{1}$, where $E_{2}$ is the kernel of $D f(x), E_{1}$ is a closed complement to $E_{2}$ in $E$ and $F_{1}$ is a complement in $F$ to the image of $D f(x)$. We may also assume

$$
f(u, v)=(g(u, v), h(u, v))
$$

for $u \in E_{1}, v \in E_{2},(u, v) \in$ domain of $f$, and where $g(u, v) \in F_{1}$ and $h(u, v) \in E_{1}$, $D h(0,0)$ is split surjective with kernel $E_{2}$ and $D g(0,0)=0$. Let $k$ be the map defined on the domain of $f$ with values in $E$ and given by $k(u, v)=(h(u, v), v)$. Then $D k(0,0)$ is an isomorphism by the closed graph theorem; as $h: E \rightarrow E_{1}$ is admissible, we have by the inverse function theorem, Theorem 3.2, for $E$ and $F$, that we may choose a neighborhood $W$ of $(0,0)$ and a $C^{1}$ diffeomorphism $\alpha=k \mid W$, so that $h \circ \alpha^{-1}(u, v)=u$ for $(u, v) \in U$; now take $V$ to be any open neighborhood of $(0,0) \in F_{1} \times E_{1}$ and $\beta$ to be the identity on $V$. Define $\eta=\beta \circ g \circ \alpha^{-1}$. We may suppose $D k(0,0)=$ identity, thus in conditions (1) and (2) if we suppose $U$ small enough, we have that $D k(u, v)$ is an isomorphism of $E$ and condition (*) in Definition 3.1 of admissibility implies $D k^{-1}(u, v)$ is locally bounded as a map from $U$ to $L(E, E)$ which implies by Proposition 2.3 that $\alpha^{-1}$ is a $C^{r}$ map. Q.E.D. 
Definition 5.1. A subset $U \subseteq W \subseteq E=\lim _{\rightarrow} E_{l}$ of an open subset of Silva space is called a chain $G_{\delta}$ when there exists $L$ so that for $l \geqslant L, U \cap E_{l}$ is a dense $G_{\delta}$ in $W \cap E_{l}$, that is, a countable intersection of dense open subsets.

It is easy to see that

Proposition 5.1. A chain $G_{\delta}$ in $W$ is dense in $W$.

THEOREM 5.2. Let $E$ be a Silva space and let $U \subseteq E$ be an open set. Suppose that $X$ is a finite dimensional second countable $C^{r}$ n-manifold, $Y$ a $C^{r}$ manifold, $\rho: U \rightarrow$ $C^{r}(X, Y)$ a $C^{r}$ representation in the sense of Abraham, $W \subseteq Y$ a submanifold of finite codimension $q$, and $\mathrm{ev}_{\rho}: U \times X \rightarrow Y$ the evaluation map. Define $U_{W} \subseteq U$ by

$$
U_{W}=\left\{a \in U: \rho_{a} \pitchfork W\right\}
$$

where $\rho_{a} \pitchfork W$ means that $\rho$ is transversal to $W$ (see Abraham-Robbin [1]). Suppose $r \geqslant n-q$. If $\mathrm{ev}_{\rho} \mid\left(\left(U \cap E_{l}\right) \times X\right) \pitchfork W$ for $l \geqslant l_{0}$ for some $l_{o}$, then $U_{W}$ is a chain $G_{\delta}$ in $U$.

To prove the above theorem we shall use the following lemmas.

Lemma 5.1. Let $F$ and $G$ be Banach spaces, $\operatorname{dim} G=n, p_{1}: F \times G \rightarrow F$ the projection on the first factor, and $E \subset F \times G$ a closed subspace of codimension $q$. If $p$ : $E \rightarrow F$ is the restriction of $p_{1}$ to $E, p=p_{1} \mid E$, then $p$ is a Fredholm operator with index $n-q$.

Proof. See [1, pp. 48-49].

LEMMA 5.2. Let $X$ and $Y$ be $C^{r}$ manifolds modelled on Silva spaces with $X$ Lindelöf. Suppose

$$
X \text { is modelled on } \lim _{\rightarrow} E_{l}=E,
$$

and suppose there exist $C^{r}$ manifolds $X_{l}$ modelled on $E_{l}$ so that $\lim X_{l}=X$ with the canonical map $X_{l} \rightarrow X_{l+1} C^{r}$. Suppose that $Y$ too satisfies $(*)$ with respect to $\lim _{\rightarrow} F_{l}=F$ and $\lim Y_{l}=Y$. Now suppose that $f: X \rightarrow Y$ is an admissible $C^{r}$ Fredholm map, and set $f_{l}=f \mid X_{l}$. Suppose

(1) $f_{l}\left(X_{l}\right) \subseteq Y_{l}$,

(2) $f_{l}^{-1}\left(Y_{l}\right)=X_{l}$,

(3) there exists $l_{0}$ so that for $l \geqslant l_{0}, f_{l}: X_{l} \rightarrow Y_{l}$ is a $C^{r}$ Fredholm map with $\operatorname{index}\left(T_{x} f_{l}\right)=\operatorname{index}\left(T_{x} f\right)$ for every $x \in X_{l}$. (Note that we do not suppose $X_{l}$ Lindelöf for any l.)

Suppose in addition for $l \geqslant l_{0}$ that if $y$ is a regular value of $f_{l}: X_{l} \rightarrow Y_{l}$, then $y$ is a regular value of $f: X \rightarrow Y$. Then the regular values of $f$ form a chain $G_{\delta}$ in $Y$.

Proof. Suppose $E=\lim _{l} E_{l}$ and $F=\lim _{l} F_{l}$ are Silva spaces and $X \subseteq E$ open. A map $f: X \rightarrow F$ so that $f \mid \vec{X} \cap E_{l} \subseteq F_{l}$ will be called chain locally closed when given any $x \in X$ there exists a neighborhood $U$ of $x$ and an integer $l_{0}$ so that for $A \subset \bar{U} \cap E_{l}$ closed in $E_{l}$ we have $f(A)$ closed in $F_{l}$, for any $l \geqslant l_{0}$. Firstly, let us show 
that $f$ is a chain locally closed map. By Theorem 5.1 above $f$ may be represented in a local coordinate system by

$$
\tilde{f}: U=U_{1} \times U_{2} \rightarrow R^{p} \times G,
$$

where $U_{1} \subset R^{n}$ and $U_{2} \subset G$ are open subsets and for $x \in U_{1}, e \in U_{2}$,

$$
\tilde{f}(x, e)=(\eta(x, e), e),
$$

where $n=\operatorname{dim} \operatorname{ker} D f(x)$ and $p=\operatorname{dim}$ coker $D f(x)$. Suppose $U_{2}=\cup_{k>0}\left(\sum_{l=1}^{k} B_{l}^{\varepsilon_{l}}\right)$, $0<\varepsilon_{l}<\infty$, and suppose $U_{1}$ a ball of finite radius, let $V_{1} \subset R^{n}$ be an open neighborhood of $x$ so that $\bar{V}_{1} \subset U_{1}$ and suppose $V_{2}$ an open neighborhood of $e$ so that $\bar{V}_{2} \subset U_{2}$. (Let us recall that a Hausdorff topological vector space is regular.) Suppose $A \subset\left(\bar{V}_{1} \times \bar{V}_{2}\right) \cap X_{l}$ is closed in $X_{l}$, and suppose

$$
(y, e)=\lim _{i} f_{l}\left(x_{i}, e_{i}\right)=f_{l}\left(\lim _{i} X_{l}\left(x_{i}, e_{i}\right)\right) \text { for } l \geqslant l_{0} \text {, when } \lim _{i} X_{l}\left(x_{i}, e_{i}\right) \text { exists. }
$$

Now $e=\lim _{i} e_{i} \in \bar{V}_{2}$ and we may assume $x=\lim _{i} x_{i} \in \bar{V}_{1}$ since $\bar{V}_{1}$ is compact. $A$ being closed we have $(x, e) \in A$ and $(y, e)=f(x, e) \in f(A)$. It is known that $\Re_{f}\left(U \cap X_{l}\right) \subseteq Y_{l}$ is dense (see [1, p. 42]).

It is an easy consequence of Theorem 3.2 that the set of critical points of a Fredholm map $f: X \rightarrow Y$ locally satisfying the hypotheses of Theorem 5.2 is closed. Thus, there exists a neighborhood of an arbitrary point $x, Z$, in $X$ so that $\Re_{f_{l}}\left(Z \cap X_{l}\right)$ is open and dense in $Y_{l}$, for $l \geqslant l_{0}$. As $X$ is Lindelöf a countable number of such $Z$ 's cover $X$; as $Y_{l}$ is a Banach manifold $\Re_{f_{l}}\left(X_{l}\right)$ is dense in $Y_{l}$. Since for $l^{\prime} \geqslant l \geqslant l_{0}$ we have $\mathcal{R}_{f_{l}}\left(X_{l}\right) \subseteq R_{f_{l^{\prime}}}\left(X_{l^{\prime}}\right)$ we obtain our result. Q.E.D.

REMARK. $\Re_{f_{l}}\left(X_{l}\right)$ is residual in $Y_{l}$.

Under the above hypothesis using an easy analogue of Theorem 5.1 and the corollary to Proposition 3.1 we can prove as in the now classical case,

Proposition 5.2. Let $X, Y$ be $C^{r}(r \geqslant 2)$ manifolds modelled on Silva spaces, $f$ : $X \rightarrow Y$ a $C^{r}$ map, $W \subset Y$ a $C^{r}$ manifold of finite codimension $q$. Then if $f \pitchfork W$, we have $f^{-1}(W)$ is a $C^{r}$ submanifold of $X$ of codimension $q$ and

$$
\left(T_{x} f^{-1}\right)\left(T_{y} W\right)=T_{x}\left(f^{-1}(W)\right) .
$$

Proof of Theorem 5.2. We shall make the simplifying assumption that $l_{0}=1$ of codimension $q$; in fact, $\mathscr{B}_{l}=\mathscr{B}_{\cap} \cap\left(U \cap E_{l}\right) \times X$ is a submanifold of $\left(U \cap E_{l}\right) \times X$. Define $\mathscr{B}=\mathrm{ev}_{\rho}^{-1}(W) \subseteq U \times X$. From Proposition 5.2 $\mathscr{B}$ is a submanifold of $U \times X$ of codimension $q=\left(\mathrm{ev}_{\rho} \mid\left(U \cap E_{l} \times X\right)\right)^{-1}(W)$ for all $l \geqslant 1$; and $U=\underset{\rightarrow}{\lim }\left(U \cap E_{l}\right)$. We shall now show that $U_{W} \cap E_{l}=R_{\Pi_{l}}$, where $\Pi: \Re \rightarrow U$ is given by the canonical projection. Suppose $a \in R_{\Pi_{l}}$ and let $y=\rho_{a}(x)$. Given $\eta \in T_{y} Y$, since $\operatorname{ev}_{\rho} \pitchfork W$ there exists $a \in T_{y} W$ and $(\alpha, \xi) \in T_{(a, x)}(U \times X)$ such that

$$
\eta=\omega+\left(T_{(a, x)} \mathrm{ev}_{\rho}\right)(\alpha, \xi)=\omega+\left(T_{1(a, x)} \cdot \mathrm{ev}_{\rho}\right) \alpha+\left(T_{x} \rho_{x}\right) \xi
$$

where $T_{1(a, x)} \mathrm{ev}_{\rho}$ is the differential of the map $a \rightarrow \rho(a, x)$. Since $\left(T_{(a, x)} \Pi\right): T_{(a, x)} \Re \rightarrow$ $T_{a} U$ is onto, let $\beta \in T_{(a, x)} \mathscr{B}$ be so that $\left(T_{(a, x)} \Pi\right)(\beta)=\alpha$. Now $\beta=\left(\alpha_{1}, \xi_{1}\right) \in T_{(a, x)} \beta$ $\cap T_{a} U \times T_{x} X$ for some $\alpha_{1} \in T_{a} U$ and $\xi_{1} \in T_{x} X$, but $\left(T_{(a, x)} \mathrm{ev}\right) \beta=\left(T_{1(a, x)} \mathrm{ev} \rho\right)(\alpha)+$ $\left(T_{x} \rho_{a}\right) \xi_{1}$ with $\left(T_{(a, x)} \mathrm{ev}\right) \beta \in T_{y} W$ which implies $\left(T_{1(a, x)} \mathrm{ev}_{\rho}\right)\left(\alpha_{1}\right) \in T_{y} W+\left(T_{x} \rho_{a}\right) T_{x} X$ so that (*) implies $\rho_{a} \pitchfork W$; hence $R_{\Pi_{l}} \subseteq U_{W}$. 
Now suppose $a \in U_{W} \cap E_{l}$, and let $x \in X$ be so that $(a, x) \in \mathscr{B}_{l}=\mathrm{ev}_{\rho}^{-1}(W) \cap$ $\left(U \cap E_{l} \times X\right)$. Since $\rho_{a} \pitchfork W$ there exists $\omega \in T_{y} W$ (where $\left.y=\rho_{a}(x)\right)$ and $\xi \in T_{x} X$ so that

$$
\left(T_{1(a, x)} \mathrm{ev}_{\rho}\right) \alpha=\left(T_{x} \rho_{a}\right) \xi+\omega, \quad \text { for } \alpha \in T_{n}(U)
$$

thus $T_{(a, x)} \mathrm{ev}_{\rho}(\alpha,-\xi)=\omega$ so that

$$
(\alpha,-\xi) \in\left(T_{(a, x)} \mathrm{ev}_{\rho}\right)^{-1} T_{y} W=T_{(a, x)} \Re_{l}
$$

and $\alpha \in\left(T_{(a, x)} \Pi\right) T_{(a, x)} \Re_{l}$, thus $U_{W} \cap E_{l} \subseteq \Re_{\Pi_{l}}$.

To prove the theorem it suffices to prove that $\Pi$ satisfies the other hypotheses of the preceding lemma. Most of these hypotheses are obviously satisfied since $\Pi$ is given by a projection of a Cartesian product. It remains only to verify that $\Pi$ satisfies the hypotheses of Theorem 5.1 locally. From Lemma 5.1 it follows that $\Pi$ : $\mathscr{B} \rightarrow U$ is a Fredholm map of index $n-q$. Now $\mathscr{B}$ is locally the inverse image of a point, say, $0 \in R^{q}$ under a submersion $f: U=U_{1} \times U_{2} \rightarrow R_{q}$ where $U_{1} \subseteq U$ and $U_{2} \subseteq X$ are open, $U_{2}$ taken to be a chart.

If $f\left(x_{0}\right)=0$ then $x_{0}$ is contained in an open neighborhood $W$ in $U$ of the form $U_{0} \times R^{q}$ where $U_{0}$ is open in $\operatorname{ker}\left(T_{x_{0}} f\right)$. Define

$$
k\left(U_{0}, \alpha\right)=\left(U_{0}, f\left(U_{0}, \alpha\right)\right)
$$

$k$ clearly satisfies the hypotheses of Theorem 5.1. $f$ is a submersion into a finite dimensional space; thus the inverse $\beta: U_{0} \times V \rightarrow U$ is a $C^{r}$ admissible map by Theorem 3.2. Thus $\beta(u, 0)$ is an admissible map. Q.E.D.

6. Some generic properties of analytic diffeomorphisms. In this section our purpose is to prove a real analytic version of the Kupka-Smale theorem for the Van Hove-Silva topology or $C^{\omega}$ topology on $\operatorname{Diff}^{\omega}(M)$, where $M$ is a compact, connected real analytic manifold.

Definition 6.1. Let $E=\lim _{n} E_{n}$ be a Silva space. A sequence $x_{n}$ in $E$ is said to strongly converge to $x \in E$ when there exists an integer $m$ so that $x_{n} \in E_{m}$ for all $n$ and the sequence converges to $x \in E_{m}$; analogously, we say that a filter $\mathscr{F}$ strongly converges to $x$ when the filter $\Phi=\mathscr{F}-x$ has the property that for the unit ball $\mathscr{B}_{m}^{1} \subseteq E_{m}$, for some $m$, we have

$$
\Re \mathscr{B}_{m}^{\prime}=\left\{\lambda \mathscr{B}_{m}^{1}, \lambda \in R\right\} \subseteq \Phi .
$$

Proposition 6.1. It is easy to see that if $x_{n}$ strongly converges to $x$ and $y_{n}$ strongly converges to $y$ to $\operatorname{Diff}^{\omega}(M)$, then $x_{n} \cdot y_{n}$ and $x_{n}^{-1}$ strongly converge to $x \cdot y$ and $x^{-1}$ respectively.

Lemma 6.1. Let $U \subseteq R^{n}$ be an open connected neighborhood of 0 of finite radius, and let $f_{n} \in C_{b}^{\omega}\left(U, R^{n}\right)$ strongly converge to $f \in C_{b}^{\omega}\left(U, R^{n}\right)$. Suppose

(1) $f_{n}(0)=0$ for all $n$,

(2) $\lim _{n} x_{n}=0, x_{n} \neq 0$, for all $n$ and $f_{n}\left(x_{n}\right)=x_{n}$.

Then 1 is an eigenvalue of $D f(0)$. 
Proof.

$$
\begin{aligned}
x_{n} & =D f_{n}(0) \cdot x_{n}+1 / 2 D^{2} f_{n}\left(t x_{n} ; x_{n}, x_{n}\right) \\
& =D f(0) \cdot x_{n}+1 / 2 D^{2} f\left(t x_{n} ; x_{n}, x_{n}\right)+A_{n} \cdot x_{n}+B_{n}\left(x_{n}, x_{n}\right),
\end{aligned}
$$

where $\lim _{n} A_{n}=0 \in L\left(R^{n}, R^{n}\right)$ and $\lim _{n} B_{n}=0$ in $L_{s}^{2}\left(R^{n} ; R^{n}\right)$, and $0 \leqslant t \leqslant 1$.

Suppose now 1 is not an eigenvalue of $D f(0)$, we have

$$
x_{n}=(I-D f(0))^{-1}\left[1 / 2 D^{2} f\left(t x_{n} ; x_{n}, x_{n}\right)+A_{n} \cdot x_{n}+B_{n}\left(x_{n}, x_{n}\right)\right]
$$

which easily leads to a contradiction.

Corollary. Given $f \in \operatorname{Diff}^{\omega}(M)$ let $\Pi_{k}(f)=\left\{x \in M \mid f^{l}(X)=x\right.$ for some $l \leqslant$ $k\}$. Suppose $f(x)$ has transversal fixed points. If $f_{n}$ strongly converges to $f$ and $\Pi_{k}(f) \subseteq \Pi_{k}\left(f_{n}\right)$ for each $n$; then there exists $N_{0}$ so that $\Pi_{k}\left(f_{n}\right) \equiv \Pi_{k}(f)$ for $n \geqslant N_{0}$.

Proof of Corollary. Let $\mu$ be the metric on $\mathbf{M}$ induced by the Riemannian metric, as the diffeomorphisms $f \in \operatorname{Diff}^{\omega}(M)$ so that $\Pi_{k}(f)$ consists of transversal fixed points of $f^{l}, l \leqslant k$, is an open set in $\operatorname{Diff}{ }^{\omega}(M)$ it follows that $\Pi_{k}\left(f_{n}\right)$ is finite for large $n$ and that $\max _{x_{i} \in \Pi_{k}\left(f_{n}\right)}\left\{\operatorname{dist}\left(x_{i}, \Pi_{k}(f)\right)\right\}$ tends to 0 . Now applying the above proposition we obtain the corollary.

Definition 6.2. A property, $P$, of analytic diffeomorphisms will be called generic when the set of diffeomorphisms, $\Omega(P) \subseteq \operatorname{Diff}^{\omega}(M)$, which satisfy $P$ is locally a chain $G_{\delta}$; that is, given any chart $\zeta: U \rightarrow \operatorname{Diff}^{\omega}(M), \zeta^{-1}(\Omega(P) \cap \zeta(U)) \subset U$ is a chain $G_{\delta}$.

In this section our objective is to prove

THEOREM 6.1. The following properties are generic.

(1) All periodic points are hyperbolic.

(2) The stable manifold, $W^{s}(p)$, and unstable manifold, $W^{u}(p)$, are injectively immersed in $M$, where $p$ and $q$ are periodic points.

(3) $W^{s}(p) \pitchfork W^{u}(q)$ for any two periodic points.

PROOF. The proof is by induction on the order of the periodic points.

Let $\tilde{P}_{n}$ (resp. $P_{n}$ ) be the collection of diffeomorphisms so that if $f^{k}(p)=p$ for $k \leqslant n, p \in M$, then $p$ is a transversal (resp. hyperbolic) periodic point; that is, the mapping $\tilde{f}(x)=\left(x, f^{k}(x)\right)$ is transversal to the diagonal $\Delta \subseteq M \times M$ at $p, f \pitchfork_{p} \Delta$. The $P_{n}$ and $\tilde{P}_{n}$ are open since the topology on $\operatorname{Diff}^{\omega}(M)$ is finer than the topologies induced by $\operatorname{Diff}^{r}(M), r \leqslant \infty$. To prove that $\tilde{P}_{1}$ is dense it suffices to show that hypotheses of Theorem 5.2 are valid for the analytic representation given by $f \rightarrow \tilde{f}$, $\operatorname{Diff}^{\omega}(M) \rightarrow C^{\omega}(M, M \times M)$, where $\tilde{f}(x)=(x, f(x))$; that is, we must show that $\operatorname{ev}_{\rho}\left(U \cap E_{l}\right) \pitchfork \Delta$ for $l \geqslant l_{0}$ for some $l_{0}$, where $U$ is a chart at $f$ and $E_{l} \equiv\left\{X \in \Gamma^{\omega}(M)\right.$, $\left\|D^{n} X\right\| \leqslant n ! M l^{n}$ for some positive constant $\left.M\right\}, \Gamma^{\omega}(M)$ being the analytic vector fields on $M$. It suffices to observe that given an $\alpha \in T_{p} M$, there exists an analytic vector field $X$ so that $X(p)=\alpha$. To see this suppose that $M$ is a submanifold of $R^{N}$ and let $A(x)=\alpha, x \in R^{N}$, be the constant vector field. By means of orthogonal projection along the normal bundle onto $T M$ we obtain an analytic vector field on $M$ so that $X(p)=\alpha$. Note that $l_{0}$ will now depend on the embedding of $M$ into $R^{N}$, but will always exist. This shows that $\tilde{P}_{1}$ is open and dense in $\operatorname{Diff}^{\omega}(M)$. 
Now we shall show that $\tilde{P}_{n} \cap P_{n-1}$ is dense in $P_{n-1}$. To do this we show that $\mathrm{ev}_{P}$ : $M \times P_{n-1} \rightarrow M \times M$ given by $\mathrm{ev}_{p}(x, f)=\left(x, f^{n}(x)\right)=\tilde{f}(x)$ satisfies $\mathrm{ev}_{p} \pitchfork \Delta$, where $f \in P_{n-1}$. Suppose $f^{n}(x)=x$ and suppose 1 is an eigenvalue of $D f^{n}(x)$. As $f \in P_{n-1}$ we have that $x=f^{0}(x), x_{1}=f^{1}(x), \ldots, f^{(n-1)}(x)=x_{n-1}$ are distinct. Now given any vector $\alpha \in T_{x_{0}} M$ we have seen there exists a vector field $X \in \Gamma^{\omega} M$ so that $X\left(x_{0}\right)=\alpha$. Let $\xi(x)$ be a polynomial in $R^{n}$ so that $\xi\left(x_{0}\right)=1$ and $\xi\left(x_{i}\right)=0$ for $0<i<n$. Consider the vector field $Y(x)=\xi(x) X(x) ; Y(x)$ determines a vector field in $E_{l}\left(\Gamma^{\omega} M\right), l \geqslant l_{0}$. For $t>0$ small enough we have that $t Y(x)$ determines an element of $E_{l}\left(C_{f}^{\omega}(M, T M)\right)$ in a canonical chart $\left(U_{f}, Y_{f}\right)$ (see [5]) of $\operatorname{Diff}{ }^{\omega}(M)$ at $f$. The path $t \rightarrow \operatorname{ev}_{p}\left(x, Y_{f}(t Y(x))\right)$ determines the vector $\left(0,\left(\exp _{R}\right)_{*}(L)\right) T_{x} M \times T_{x} M$, where $\exp _{R}$ is the Riemannian exponential. This shows that the hypotheses of Theorem 5.2 are satisfied and thus $\tilde{P}_{n} \cap P_{n-1}$ is an open dense subset of $P_{n-1}$.

We shall now show that $P_{n}$ is an open dense subset of $\tilde{P}_{n} \cap P_{n-1}$. That $P_{n}$ is open is obvious for the topology on $\operatorname{Diff}^{\omega}(M)$ is finer than the topology on $\operatorname{Diff}^{r}(M)$ for any $r \leqslant 0$.

Consider $M$ a Riemannian analytic submanifold of $R^{N}$, and let $X_{x_{1}}$ be the vector field on $R^{N}$ given by $X_{x_{1}}(x)=x_{1}-x$. Suppose $p_{1}, \ldots, p_{s}$ are the points of $\Pi_{n}(f)$, for $f \in \tilde{P}_{n} \cap P_{n-1}$. Suppose that $p_{1}$ is not a hyperbolic fixed point, note that $n$ is a primitive period of $p_{1}$; that is, $n$ is the smallest positive integer so that $f^{n}\left(p_{1}\right)=p_{1}$.

Set

$$
\xi(x)=\frac{\left\|x-p_{2}\right\|^{2} \cdots\left\|x-p_{s}\right\|^{2}}{\left\|p_{1}-p_{2}\right\|^{2} \cdots\left\|p_{1}-p_{s}\right\|^{2}}
$$

and $Y_{p_{1}}(x)=\xi(x) X_{p_{1}}(x)$. Let $\eta_{p_{1}}(x)$ be the orthogonal projection onto $T M$ of the restriction of $Y_{p_{1}}$ to $M$. Suppose $\varepsilon>0$ small enough so that if $g \in \operatorname{Diff}^{\omega}(M)$ is the diffeomorphism corresponding to $\varepsilon \eta_{p_{1}}(x)$ under the canonical chart at the identity then

(i) $\Pi_{n}(g f)=\Pi_{n}(f)$,

(ii) hyperbolic fixed points of $\Pi_{n}(f) \subseteq$ hyperbolic fixed points of $\Pi_{n}(g f)$,

(iii) transversal fixed points of $\Pi_{n}(f)=$ transversal fixed points of $\Pi_{n}(g f)$,

(iv) all the fixed ponts of $g f$ are transversal, and

(v) the spectrum of $D\left((g f)^{n}\right)_{p_{1}}$ does not touch the unit circle.

It is clear that $p_{1}$ is a hyperbolic fixed point of $g f$. Thus $P_{n}$ is dense in $\tilde{P}_{n}$.

Now by construction $\psi_{f}\left(P_{n} \cap U_{f}\right)$ is a chain $G_{\delta}$; that is, there exists $l_{0}$ so that $\psi_{f}\left(P_{n} \cap U_{f}\right)$ is the countable intersection of open dense subsets, in the image of $U_{f}$ in $E_{l}\left(C_{f}(M, T M)\right)$ for $l \geqslant l_{0}$, where $C_{f}(M, T M)$ is the space of liftings of $f$ and $\psi_{f}$ is a canonical chart at $f \in \operatorname{Diff}^{\omega}(M)$. This implies that $P=\bigcap_{n} P_{n}$ is dense in $\operatorname{Diff}^{\omega}(M)$.

To show that the hypotheses of Theorem 5.2 are satisfied at a diffeomorphism $h=Y_{f}(Z)$, for $Z$ in a small enough canonical chart at $f$ we just perturb $Z$ by a small enough translation by the vector field we considered in the above proof to show transversality at $f$.

To prove (2), as in the Kupka-Smale theorem for $C^{r}, r<\infty$ (see Smale [16]), we have that the local unstable manifold theorem (see [14]) implies that the stable and unstable manifolds of an analytic diffeomorphism at a hyperbolic fixed point $p$ are analytically immersed submanifolds of $M$. Let us recall that the stable manifold is 
given by $W^{s}(p)=\cup_{n \geqslant 0} f^{-n} W_{\mathrm{loc}}^{s}(p)$, where $W_{\text {loc }}^{s}(p)$ is the local stable manifold, and the unstable manifold by $W^{u}(p)=\cup_{n \geqslant 0} f^{n}\left(W_{\text {loc }}^{u}(p)\right)$, where $W_{\text {loc }}^{u}(p)$ is the local unstable manifold.

To prove (3) note that the topology of $\operatorname{Diff}^{\omega}(M)$ being finer than the topology induced by $\operatorname{Diff}^{\infty}(M)$ implies that $D_{n}=\left\{f \in \operatorname{Diff}^{\omega}(M): p, q\right.$ periodic with period $\left.\leqslant n, W^{s}(p) \pitchfork W^{u}(q)\right\}$ is open in $\operatorname{Diff}^{\omega}(M)$.

As in the proof of (1), it suffices to suppose we are in a canonical chart at the identity $V$. Let $f \in V$ be a diffeomorphism with hyperbolic periodic points and suppose $p$ and $q$ are periodic points of $f$ with periods $\leqslant n$. Suppose for some $m<n$ we have $W_{\text {loc }}^{s}(p) \pitchfork f^{i} W_{\text {loc }}^{u}(q)$ for $i \leqslant m$ but $W_{\text {loc }}^{s}(p)$ intersects $f^{m} W_{\text {loc }}^{u}(q)$ nontransversally at $f(x)$; note that $x \notin f^{m-2} W_{\text {loc }}^{u}(q)$. Let $U$ be a neighborhood of $x$ so that

$$
\begin{aligned}
U \cap\left(W_{\mathrm{loc}}^{s}(p)\right. & \left.\cup f^{m-2} W_{\mathrm{loc}}^{u}(q)\right) f^{m-1} W_{\mathrm{loc}}^{u}(q) \\
\cup & \cdots \cup\left(f W_{\mathrm{loc}}^{u}(q) \cup W_{\mathrm{loc}}^{u}(q)\right)=\varnothing .
\end{aligned}
$$

As in the proof of (1) construct a diffeomorphism near the identity so that

(1) $\Pi_{n}(g f)=\Pi_{n}(f)$,

(2) $f \mid\left[U \cap f^{m-1} W_{\mathrm{loc}}^{u}(q)\right] \pitchfork W_{\mathrm{loc}}^{s}(p)$.

The embedding of $M$ plays an essential role here in determining $l_{0}$.

Since $D_{n, m}=\left\{f \in \bigcap_{i=1}^{n} H_{i}: \forall p, q\right.$ with period $\left.\leqslant r W_{\mathrm{loc}}^{s}(p) \pitchfork f^{i} W_{\mathrm{loc}}^{u}(q) \forall i \leqslant m\right\}$ is open and dense in $E^{l}$ for $l \geqslant l_{0}$, we have that $\bigcap_{n, m} D_{n, m}$ is a chain $G_{\delta}$.

ACKNOWLEDGEMENTS. The author thanks M. Hirsch for a critical reading of an earlier version of this work. Gratitude is due R. Palais and R. Williams for their encouragement. I had helpful conversations with J. F. Colombeau, J. Franks and C. Robinson for which it is my pleasure to thank them. Finally, I wish to thank the referee for his detailed and painstaking reading of this manuscript.

\section{BIBLIOGRAPHY}

1. R. Abraham and J. Robbin, Transversal mappings and flows, Benjamin, New York, 1967.

2. N. Bourbaki, Topologie générale, Chap. II, Hermann, Paris, 1967.

3. J. F. Colombeau, Differentiation et bornologie thèses, U.E.R. Mathématiques et Information, Université de Bordeaux, 1973.

4. __ Sur quelques particularités du calcul différentiel dans les espaces vectoriels topologiques ou bornologiques, Rev. Roumaine Math. Pures Appl. 18 (1973), 3-17.

5. J. Dieudonné, Denumerability conditions in convex vector spaces, Proc. Amer. Math. Soc. 8 (1957), $367-372$.

6. J. Hale, Theory of functional differential equations, Springer-Verlag, New York, 1977, pp. 320-333.

7. P. Hartman, Ordinary differential equations, Wiley, New York, 1964.

8. M. W. Hirsch and C. Pugh, Stable manifolds and hyperbolic sets, Proc. Sympos. Pure Math., vol. 14, Amer. Math. Soc., Providence, R. I., 1970, pp. 133-163.

9. H. Hogbe-Nlend, Théorie des bornologie et applications, Springer-Verlag, Berlin and New York, 1971.

10. L. Van Hove, Topologie des espaces fonctionels analytiques et des groupes infinis de transformations, Acad. Roy. Belg. Bull. Cl. Sci. (5) 38 (1952).

11. H. Jacobowitz, Implicit function theorems and isometric embeddings, Ann. of Math. 95 (1972), $191-225$

12. J. Leslie, On a differential structure for the group of diffeomorphisms, Topology 6 (1967), 264-271.

13. S__ Some Frobenius theorems in global analysis, J. Differential Geom. 2 (1969), 279-297.

14. D. C. Lewis, Invariant manifolds near an invariant point of unstable type, Amer. J. Math. 60 (1938), 577-589. 
15. José Sebastio e Silva, Su certe classi di spazi localmente convessi imiportanti per le applicazioni, Rend. Mat. (5) 14 (1955), 338-410.

16. S. Smale, Differential equations and diffeomorphisms, Ann. Scuola Norm. Sup. Pisa Cl. Sci. (4) 17 (1963), 97-116.

17. , Differentiable dynamical systems, Bull. Amer. Math. Soc. 73 (1967), 747-817.

18. E. Zehnder, Generalized implicit function theorems with applications and some small divisor problems, Comm. Pure Appl. Math. 28 (1975), pp. 91-140.

19. ___ Moser's implicit function theorem in the framework of analytic smoothing, Math. Ann. 219 (1976), pp. 105-121.

Department of Mathematics, NorthWESTERn UNiversity, EVANSTON, ILlinois 60201 